EUROPEAN ORGANIZATION FOR NUCLEAR RESEARCH

CERN-PPE/94-180

November 15, 1994

\title{
Construction and testing \\ of a precision 2 meters long straw chamber prototype
}

G. Bonvicini, R. Jacobsen

CERN, CH-1211 Geneva, Switzerland

E. Veitch

Dept of Phys., Univ. of Edinburgh, Edinburgh EH9 3JZ, United Kingdom

M. Bertoldi, D. Levinthal

Phys. Dept., Florida State Univ., Tallahassee FL 32306

G. Barber, W. Cameron, M. Cattaneo

Imperial College, London SW7 2BZ, United Kingdom

H. Kim, J. Rothberg

Dept. of Physics, Univ. of Washington, Seattle 98195

\begin{abstract}
The construction and testing of a 2 meter straw chamber prototype is described. We achieved straw alignment of better than $30 \mu \mathrm{m}$ and wire alignment of order $7 \mu \mathrm{m}$ while keeping the amount of material to about one half the amount normally needed for a similar all-wire drift chamber.
\end{abstract}

Submitted to: Nuclear Instruments and Methods 
Straws are uniquely versatile particle detectors. Their cheapness, ruggedness, lightness and ease of assembly make them suitable for muon detectors, central and vertex trackers, and transition radiation detectors.

In the following we report on the construction and performance of a 63 straws, 2-meters long prototype. We were particularly interested in a possible replacement of the ALEPH Inner Tracking Chamber with something having the best possible protection against broken wires (which stay in the straw, as opposed to the potential danger of massive short circuits in an open cell geometry), good alignment and twice the granularity for the same mass. The latter would improve the vertexing capabilities of ALEPH.

The prototype discussed below was designed with these ideas in mind. We found a novel way to align both straws and wires to very high precision over two meters. In Section 1 we discuss the design constraints, the mechanical problems related to detector construction and some alternative solutions. In Section 2 the chosen assembly system is described and in Section 3 the results of the cosmic ray tests are presented and discussed.

\section{Design considerations and assembly meth- ods}

If the aim is to build a chamber that is very long and very well aligned throughout, some problems are common to shorter chambers and others are due to the length of the straws. Both straws and wires need to be aligned. Straws, as extended, flexible objects, can not be aligned as precisely as wires. Sources of error on the wire positioning are:

- misalignment at the end plate

- overall misalignment w.r.t. the straw, inducing an electrostatic deflection on the wire. This source of error is strongly dependent on the straw length.

- if the electrostatic deflection is too strong, a wire support must be introduced, coupling mechanically wire and straw. The inferior alignment of the straw will then be passed onto the wire.

For this test we used straws from Ref. [1] with a wall thickness of $54 \mu \mathrm{m}$.

The average thickness seen by a particle travelling perpendicular to the tube 
axis is $0.06 \% X_{0}$ if the straw diameter is $10 \mathrm{~mm}$. Straws, as they are produced today, are remarkably uniform in circumference (to a tolerance of $15 \mu \mathrm{m}$ or better) but are neither straight nor round. After manufacturing, leftover tensions in the plastic material make an unsupported 2-meter straw deviate by a few centimeters from a straight cylinder.

An evaluation of the electrostatic deflection of a wire displaced from the axis of a straw determines the requirements for wire-straw alignment. If $\delta$ is the displacement from the center line without any applied voltage, $L$ the length of the straw, $R$ the straw internal radius, $r$ the wire radius, $V$ the voltage applied, and $T$ the wire tension, the maximum deflection of the wire due to the applied voltage will be[2]

$$
D=\frac{\pi}{2} \delta \frac{V^{2}}{V_{0}^{2}-V^{2}}
$$

where $V_{0}$ is the stability limit and

$$
V_{0}=\frac{R}{L} \log \frac{R}{r} \sqrt{\frac{\pi T}{2 \epsilon_{0}}} .
$$

For typical values of the chamber parameters,

$$
D \sim \delta, \text { if } L=2 m
$$

and

$$
D \sim 0.01 \delta \text {, if } L=67 \mathrm{~cm} .
$$

Hence, at a length of 2 meters the wire will be aligned as well as the straw is, because either the electrostatic or the mechanical coupling via a wire support will deflect it by a quantity of order $\delta$. For shorter lengths wire and straw are essentially decoupled.

We first discuss methods to align the straws. Earlier solutions to make straws perfectly cylindrical were to pull and inflate them [3]. This would, in turn, involve having thick outer shells, increasing the material unacceptably by today's standards. Our tests of the straws described below indicated that absolute pressures of order 2 atmospheres and tensions of order $1 \mathrm{~kg}$ were needed to make 2 meter long straws straight and round to $20 \mu \mathrm{m}$.

A more refined approach is the one proposed in Ref.[4]. Each straw is fitted round a precision steel rod, and the rods are held vertically in a closelypacked matrix by positioning plates at the two ends of the rods. A low-mass 
gluing method is used during assembly, and after the rods are pulled out the straw lattice forms a composite material which is strong, self-supporting, and able to withstand the axial pressure due to the wire tension. Contrary to popular belief, this arrangement has less material than an open cell, all wire, geometry can typically achieve. The axial pressure is reduced by typically one order of magnitude, because of the lack of field wires, and the material put into the straws is saved on the support shell. This method can place the straws with about $30 \mu \mathrm{m}$ accuracy, and the wires with about $20 \mu \mathrm{m}$ accuracy at the end plate.

When extrapolated to a 2-meter long detector, this method becomes rather clumsy, with tons of precision rods involved, and rods of this length tend to scratch the inside of a straw much more easily.

We tested first a variant of this method, described in Fig.1. For these tests we used Mylar-Aluminum straws, $R=4.000 \mathrm{~mm}$, and precision rods with measured diameters between 7.990 and $7.997 \mathrm{~mm}$. A massive aluminum mold provided the shape for a triangular, 21-straw array. Rods were inserted into the straws and placed into the mold which lay on a precision horizontal surface. A thin layer of viscous glue was applied at the straw-straw contact points.

After drying, the cast was removed, the rods pulled out, and the triangular array measured for alignment against a precision marble plane. The results for one such array are shown in Fig. 2. Distortions of 200-300 microns are visible in each of the measured straws. Possibly the outer straws are not supported well enough by the other straws in the lattice, or the whole module has some distortions of its own.

Subsequently, two of these arrays were fully equipped as detectors, and took data in the cosmic beam setup described in Section 3. The results showed that the wires were of order 200 microns misaligned with respect to their nominal position, which is consistent with the electrostatic deflection. Next we tried an aligning matrix made of graphite[5], as shown in Fig. 3. The holes were precision-drilled, so that about $90 \%$ of the plate material was removed, the diameter of the holes into which the straws were to be inserted being $8.10 \mathrm{~mm}$. The minimum distance between two neighbouring holes (and hence two neighboring straws) was $0.45 \mathrm{~mm}$ and the plate thickness was $6.3 \mathrm{~mm}$.

A metrological measurement of the alignment of these holes, see Ref.[5], gave an RMS of $45 \mu \mathrm{m}$, which is acceptable. Graphite is a totally amorphous 
material with low heat conduction, which explains why the distortions are so low when similar drilling conditions would have macroscopically warped or destroyed a metal or carbon fiber plate.

Measurements, however, showed that such plates were needed every 20 $\mathrm{cm}$ to maintain the straws straight and hence that an unacceptable amount of support material must be incorporated.

\section{The new assembly method.}

The considerations and tests described above made it clear that several problems need be addressed.

- the straws and wires need to be aligned on the same precision plate, to minimize misalignments.

- the straws need to be constrained continuously.

- to minimize straw-wire electrostatic pull, the wire should be supported over lengths shorter than 2 meters.

We introduced several novel solutions to achieve this. To build this new prototype, we used $R=5.000 \mathrm{~mm}$ Kapton-copper straws from [1]. They had mechanical properties similar to Mylar-aluminum straws of the same dimensions.

The prototype is schematically shown in Fig. 4. Its total length was $193 \mathrm{~cm}$, and two carbon fiber membranes were placed at $64.3 \mathrm{~cm}$ from the end plates. The straws were also $64.3 \mathrm{~cm}$ long, but the wires were $193 \mathrm{~cm}$ long and were fed through the membranes.

The membranes and end plates were drilled as shown in Fig. 5. The larger holes, with a radius of $2.5000 \mathrm{~mm}$, housed the wire feed-throughs described later. The smaller holes, with a radius of $(2 / \sqrt{3}-1)$ times the straw radius (Fig. 6), were used as feed-throughs for precision-made disposable Teflon strings which were tangent to the straws along the whole length and provided the alignment constraints for the straws at the time of assembly.

The feed-through for the wires, the electric contact and the straw collars that interfaced the straws and the membranes or end plates are shown in Fig. 7. The electric contact was made with a small strip of copper, which fitted into small slots in the collar, and was glued to the inside of the straw 
by means of conductive Epoxy. The copper strip was fed across the end plate or membrane through small notches in the wire feed-through (Fig. 8). The collar made the straw round, flat and hard at the point of mechanical junction with the end plates or membranes, to which it was glued by a single drop of glue. Its funnel-like shape helped considerably while stringing the wires.

The feed-through is a simple hard-plastic, molded cylinder, with a precision $\mathrm{V}$ which positions the wire (Fig. 8). The $\mathrm{V}$ only provides a constraint in one direction, and if electrostatic forces on the wire have a component pointing away from the $\mathrm{V}$, the wire will be effectively unconstrained. To overcome this problem, the two membranes were positioned $2 \mathrm{~mm}$ higher than the end plates, with the $\mathrm{V}$ pointing down (Fig. 9). The wire tension then had a positive component pushing the wire into the V. A simple calculation showed that a straw-wire misalignment of $50 \mu \mathrm{m}$ (the estimated tolerance) would have caused the wire to lift off the $\mathrm{V}$ at a voltage of $30 \mathrm{kV}$.

The carbon fiber end plates and membranes have shown exceptional properties, which some of us reported separately one year ago [5]. Since then, "isotropic carbon fiber" plates have become the end plates of choice at all low-energy particle physics factories [6, 7]. Briefly, carbon fiber with the fiber planes laid in a simple isotropic pattern is about one order of magnitude more precise than metal plates, (we measured $2-3 \mu \mathrm{m}$ accuracy for our membranes, $5-7 \mu \mathrm{m}$ for our plates, and $23 \mu \mathrm{m}$ for the control metal plates) and one order of magnitude lighter. The accuracy error is dominated by short range imperfections and not by overall distortions of the plate or membrane [5]. The 2-dimensional position of each $\mathrm{V}$ in the plate or membrane was measured with an accuracy of approximately 2 microns.

We used $6.5 \mathrm{~mm}$ thick plates and $0.5 \mathrm{~mm}$ thick membranes. The material around the membrane region is composed mostly of plastics, and contributes no more than $1.0 \% X_{0}$ for a particle crossing the membrane at $45^{\circ}$ from the detector axis.

As shown in Fig. 4, the prototype was mounted on a machined I-beam, which allowed support and ease of handling. The assembly was performed vertically. Two extra plates, made of $5 \mathrm{~mm}$ thick aluminum, were drilled with the hole pattern of Fig. 5. and then bolted at the extremities of the I-beam. Their role was to take the tension of the strung Teflon wires during assembly.

A first layer of Teflon wires made by [1] was strung, with a tension of $1 \mathrm{~kg}$, and crimped at the two metal plates. The first layer of straws was 
then added, electrically connected to the continuing straws, glued to the end plates and membranes ${ }^{1}$, and glued to the adjacent straws. For straw-straw gluing, a single drop of glue was applied every $7 \mathrm{~cm}$. The glue then would propagate along the crack between two straws, covering all of the intervening space.

The surface tension of the glue was significantly stronger than the force needed to restore straightness in a straw (which was of order 0.2 grams). The strings under tension were 6 times stronger than straws with respect to lateral forces. With glue, straight strings, and other uniform straws on every side, each straw was subject to relatively large constraints in each direction. The glue surface tension effectively exerted the same lattice-ordering strength everywhere, contributing to the uniformity of the lattice. The surface tension did not depend on the quantity of glue, as small excesses would run down the straw. The tolerance between straw and string diameters, and the spacing of the lattice, was 30 microns.

The assembly proceeded with alternate layers of strings and straws until completion. The body of the prototype, comprising a total of 63 straws, took 24 hours for two people to complete. The glue drying time was 36 hours.

After drying, the no-stick Teflon strings were removed. Both ends were pulled at the same time, and as the Teflon string stretched, it became thinner, so that when one crimp had been cut the whole string could be recovered at the other side. The body of the detector was then extremely well aligned with no extraneous material other than in the membrane region.

To estimate the straw alignment, we built some modules of one layer only using spare end plates, and then measured the linearity of the straw-straw gap (visible because of the glue transparency), in a vertical position, with respect to a $100 \mu \mathrm{m}$ steel wire under $1 \mathrm{~kg}$ tension. The straw-straw gap could have been as large as $1 \mathrm{~mm}$ before gluing but the straws would be pulled together by the glue, which was effectively acting like a zipper. All gaps then were the same to roughly $15 \mu \mathrm{m}$, and all were roughly $30 \mu \mathrm{m}$ wide (Fig. 10a)). The linearity of the straw-straw gap is shown in Fig. 10b), with an average deviation of less than $30 \mu \mathrm{m}$. Finally, the profile of a straw in the direction perpendicular to the straw plane is shown in Fig. 10c), and is one order of magnitude worse than in the straw plane direction, giving a rough estimate of how much alignment is gained from immediately adjacent strings and how

\footnotetext{
${ }^{1}$ Throughout the construction we used the glue of Ref.[8] for all gluing operation.
} 
much from the whole lattice. We conclude that the straws in our prototype were aligned and cylindrical to better than $30 \mu \mathrm{m}$ along their length.

Wire stringing was also done vertically. 20 micron thick gold-plated tungsten wire was dropped through the straw with a needle as lead, and strung with a tension of 53 grams. The crimps were slightly off-center so that the wire would be positively pulled into the end plates feed-through V. Stringing took 24 more man-hours.

Before transportation to the data-taking site, the whole prototype was fitted with end cans with wire and gas feed-throughs, and a plexyglass encasing to contain the gas and to protect against damage.

\section{Data taking and results.}

Cosmic ray data were taken using the set-up of Fig. 11 . The trigger and $t_{0}$ were given respectively by the coincidence and OR of the two scintillators, and the two multiwire chambers provided a $2-D$ track coordinate measurement. About $1.4 \times 10^{5}$ cosmic triggers were taken with this set-up, over 20 runs of one day each. All runs were taken with a gas mixture of $89 \%$ Argon, $10 \%$ carbon dioxide, and 1\% methane (HRS gas).

17 of the 20 runs were taken at a HV of $1425 \mathrm{~V}, 12 \mathrm{~cm}$ from a membrane and $52 \mathrm{~cm}$ from an end plate. One run was taken at a $\mathrm{HV}$ of $1325 \mathrm{~V}$ and one run at a $\mathrm{HV}$ of $1375 \mathrm{~V}$. One run was taken at $1425 \mathrm{~V}$ in mid-span between the membrane and end plate. The latter three runs were taken for cross checks, showed little change of the resolution or efficiency with high voltage and showed that the wires were in the expected position in mid-span. They were not used for the following analysis.

Only 32 amplifier channels were available, later reduced to 30 , and they were connected according to Fig. 12. The electronics and readout system, including the software, were as used by the ITC in ALEPH and are described in Ref. [9]. Briefly, the hardware consists of a preamplifier ( $\times 3$ in voltage), connected to a postamp-discriminator $(\times 36$ in voltage $)$, sitting in a large format $(9 \mathrm{U})$ Euro crate. The discriminator outputs are connected to a Lecroy 4290 TDC system, with 0.5 nsec timing resolution, which provides for a hardware timing calibration which was run approximately weekly.

The analysis used the metrology information on the position ${ }^{2}$ of the V's

\footnotetext{
${ }^{2}$ For a discussion of the metrology measurements on membranes and end plates, see
} 
and the position of the wire was assumed to be a weighted average between the end plate wire position and the membrane wire position (this correction was typically only a few microns).

A typical event is shown in Fig. 13. Data were analyzed in three steps. A first step would select usable tracks, fit them for all possible left-right combinations, and select the best, assuming the nominal wire positions. A fit for the linear time-to-distance coefficient giving the best possible resolution was then performed. A second fit allowed for small, quadratic deviations from linearity, and hits with a residuals larger than $0.8 \mathrm{~mm}$ were discarded.

The efficiency was measured as follows. Given four straws that fit to a good track, other intermediate straws were looked at to see whether they had fired. The efficiency was found to be $96.0 \%$. If a cut was applied such that the fitted track was at least $1 \mathrm{~mm}$ inside the straw under study, the efficiency was found to be $98.7 \%$, which suggests that most of the inefficiency is purely geometrical.

To measure the resolution we considered again the same five-hit tracks, and measured the residual of each straw with respect to the fit using the other four. Simple algebra shows that the width of this distribution is the spatial resolution multiplied by a factor $\sqrt{3 / 2}$ if the straw is the first or last of the five, and a factor $\sqrt{5 / 4}$ if the straw is the middle one. The resolution was measured to be $180 \mu \mathrm{m}$ (Fig. 14), with all measured wires within $7 \%$ of this value, which is consistent with the well-known resolution of HRS gas, and was found to depend very little on the voltage in use. Unfortunately, safety considerations prevented the use of more precise gases, such as dymethilether, which would have constrained the alignment even better.

All hits with a measured distance from the wire larger than $1 \mathrm{~mm}$, on tracks with at least five hits, were then used to measure the position of the wire, assuming straight cosmic tracks. The fitted gaussian peak was used, rather than the average of the hits, to produce the results, as some asymmetry was present in the tails. The difference between the metrology-measured $x-$ coordinates and the track-measured $x$-coordinates are shown, for each wire, in Fig. 15. The errors fluctuate because of acceptance, being largest for straws on the outside in the middle planes, and smallest on the inside in the upper or lower planes.

There is an overall offset, and two degrees of freedom for the time-toRef.[5]. 
distance relationship, leaving a total of 27 degrees of freedom. The total $\chi^{2}$, defined as the sum of all the differences, divided by the fitted $\sigma$, and squared, is 67 , which shows that there is some residual misalignment. We estimate the residual misalignment by adding in quadrature a systematic error, and imposing that the $\chi^{2} /$ dof be equal to 1 . We then estimate the error by a variation of 1 in the total $\chi^{2}$. The result is that the data are consistent with a misalignment of $7.0 \mu \mathrm{m}$. The error on this quantity is estimated to be of order 1 micron.

Possible causes for this relatively large misalignment could be related to the positioning $\mathrm{V}$. First, the $\mathrm{V}$ had an angle of $120^{\circ}$, which might not be adequately forcing the wire to the apex. Perhaps an angle of $90^{\circ}$ would have been better. Second, the edges of the V's were rough, because of the plastic that was used. The position of the $V$ was measured by taking three points on either $\mathrm{V}$ leg, and fitting the apex position. Possibly the roughness of the legs was enough to offset the $2 \mu \mathrm{m}$ error of the metrological measurement. Finally, the use of the fitted resolutions instead of the mean squared deviations might have slightly overestimated the misalignment contribution, but we have estimated this effect to be small.

\section{Conclusions.}

We have built a 2-meter long, 63-straws prototype aligned to within $7 \mu \mathrm{m}$. Such accuracy was achieved with amounts of material which are less than, or of order of, those commonly used in trigger chambers. The relevant method involved the use of very precise carbon fiber end plates and membranes, for wire and straw positioning, and a special assembly method to align the straws throughout.

In retrospect, the straws are so well aligned by this method that probably detectors of less than $120 \mathrm{~cm}$ of length could achieve a wire alignment of 30 microns or better without any intermediate support. Thus, trigger chambers at low energy factories could use this technology for minimal mass, high precision detectors.

We would like to thank Mike Price and C. Rivoiron for many discussions, B. Brunel, P. Charra, R. Currat and R. Poncet for technical help, C. Davis and D. Baxter for extensive testing of the straws and collar and feedthroughs moulding, P. Schilly for the test beam setup, R. Angelloz for the metrology 
work, S. and P. Hester for technical assistance with the straws and Teflon strings. J. Wotschack, J. Otto, and D. Balboni contributed to the early part of this effort. Best thanks to H. Kagan for introducing us to the straw tubes technique. 


\section{References}

[1] Lamina Dielectrics, Myrtle Lane, Billinghurst - West Sussex RH149SG, United Kingdom.

[2] J. Carr and H. Kagan in the Proceedings of the 1986 DPF Summer Study on Physics of the Superconducting Supercollider, Snowmass, Colorado (1986) p.396.

[3] See, for example, W.W. Ash et al., Nucl. Instr. and Methods, 261, 399, 1987.

[4] M. Frautschi et al., Nucl. Inst. and Methods, 307, 52, 1991.

[5] For a detailed description of the graphite and carbon fiber plates, including providing companies, see: G. Bonvicini, M. Bertoldi and H. Betzold, CERN-PPE-93-15.

[6] BABAR Proposal, SLAC-443.

[7] R. Baldini et al., LNF-93-002 and LNF-94-028.

[8] The glue used throughout was a $33-67 \%$ mixture of Epon 828 catalyst from Shell Corporation, P.O. Box 1380, Houston TX 77251, USA, and standard Araldite D, from CERN. The glue was very liquid and penetrating and dried to the consistency of hard rubber.

[9] Aleph Handbook, ALEPH 89-77.

\section{Figure Captions}

1. Schematic representation of the first method tried. The straws, containing precision rods, are packed into a massive, precision aluminum form for gluing. The drawing shows 3 layers and six straws. The real method involved 6 layers and 21 straws.

2. Measured profiles of the outer straws, versus position along the straw. The scale is indicated by a bar in each Figure. a) horizontal profile. b) vertical profile. 
3. Schematic representation of a graphite plate. The holes were precisiondrilled with about $90 \%$ of the material being removed.

4. Schematic representation of the prototype. The body of straws is divided in three sections, but the wires go through the membranes and are crimped at the end plates. Visible are the I-beam support, to which the end plates and membranes are connected by aluminum frames. Not shown are the heavy aluminum plates at the ends of the I-beam, which were used to support the tension of the Teflon strings during assembly.

5. Hole pattern of membranes and end plates. The larger holes are the feed-throughs for the wire and contain a wire positioning precision $\mathrm{V}$. The smaller holes are for the precision Teflon strings used at the time of assembly.

6. How the straws and the Teflon strings fit together. Each internal Teflon wire is surrounded by three straws, and each straw is surrounded by six strings. The required tolerance was $30 \mu \mathrm{m}$.

7. Material around the membrane region. Shown are the straw collars, made of hard plastic and $0.5 \mathrm{~cm}$ high, the copper electric contact, and the wire feed-through.

8. The precision feed-through. a) side view. b) front view.

9. Orientation of each segment in the vertical plane. All Vs are pointing down, and the two membranes had the hole pattern $2 \mathrm{~mm}$ higher than the end plates.

10. a) Straw-straw gap profile. The gap was uniform to within $15 \mu m$ (the sensitivity of our optical measurement). b)straw-straw gap linearity with respect to a steel wire under tension. Some distortion is visible. c) Straw profile in the direction perpendicular to the straw array. The distortion is about ten times larger than in the plane of the array (previous Figure), showing the importance of the straw lattice in aligning single straws.

11. Cosmic rays setup. Two photomultipliers and two $10 \times 10 \mathrm{~cm}^{2}$ chambers provided the external hodoscope. 
12. Straw tubes connected to the electronics.

13. A cosmic ray event. The circles give the distance from the wire as measured by the detector.

14. Single-hit resolution, $H V=1425 \mathrm{~V}$, for one straw in the first layer from bottom.

15. Difference between the $x$-coordinate, as measured optically, and the $x$ - coordinate as measured with the tracks, for each wire. 